\title{
Avaliação contínua da aprendizagem por meio da aplicação de quiz semanal no ensino da Periodontia
}

\author{
João Paulo Steffens*; Stephanie von Stein Cubas Warnavin**; Humberto Osvaldo Schwartz Filho*; \\ Geisla Mary Silva Soares*; Ângela Fernandes*; Edison Luiz Almeida Tizzot*** \\ * PhD, Departamento de Estomatologia, UFPR \\ ** Mestranda, Programa de Pós-Graduação em Odontologia, UFPR \\ *** $\mathrm{PhD}$, Departamento de Tocoginecologia, UFPR
}

Recebido em 01/03/2018. Aprovado em 21/09/2018.

\begin{abstract}
RESUMO
A avaliação contínua e rotineira mantém o estudante sob constante estudo, leitura e aprendizado, além de suavizar o impacto das avaliações teóricas formais sobre os critérios tradicionais de aprovação. O objetivo deste estudo foi avaliar o impacto da aplicação de quiz semanal sobre a nota da avaliação teórica formal no componente curricular Periodontia I do Curso de Odontologia da Universidade Federal do Paraná. Para este estudo observacional retrospectivo, avaliaram-se os campos "nota quiz" e "nota avaliação" dos diários de classe das turmas do primeiro e segundo semestres de 2016, respectivamente "sem quiz" (n=53) e "com a realização de quiz semanal" (n=55). As avaliações teóricas formais foram idênticas para as duas turmas. Os dados referentes ao número de quizzes realizados e média das notas de quiz foram tabulados. As análises estatísticas utilizadas foram teste $t$ de Student e coeficiente de correlação de Spearman. A aplicação de quiz semanal deslocou a curva referente às notas de avaliação teórica à direita, sendo que as notas foram significativamente maiores neste grupo $(\mathrm{p}<0.01)$. Além disso, houve correlação significativa entre a nota da avaliação teórica e o número de quizzes realizados por cada estudante $(r=0,39 ; \mathrm{p}<0,01)$, bem como com a média da nota do quiz $(\mathrm{r}=0,47 ; \mathrm{p}<0,01)$. Concluiu-se que a utilização de quiz semanal como instrumento de avaliação da aprendizagem influi positivamente no grau de conhecimento adquirido, provavelmente devido a um maior estímulo à leitura e estudo constante.

Descritores: Avaliação Educacional. Aprendizagem. Periodontia. Estudo Observacional. Educação em Odontologia.
\end{abstract}

\section{INTRODUÇÃO}

A avaliação no Ensino Superior deve ocorrer durante todo o processo de ensino, incluindo as relações dinâmicas entre docente e estudante nos ambientes de aprendizagem, e deve orientar as decisões relacionadas ao conhecimento $^{1}$. Assim, o processo avaliativo desvela a competência do educador e a adequação da proposta ao projeto pedagógico ${ }^{2}$. De fato, as formas tradicionais de avaliação no 
ensino superior são questionadas à medida que não refletem fielmente a dedicação e empenho do estudante de forma contínua, sendo urgente a necessidade de novas propostas e posturas nos ambientes de aprendizagem ${ }^{3}$.

De maneira alinhada à visão dos educadores supracitados, a atual Lei de Diretrizes e Bases (Lei 9394/96) ${ }^{4}$, em seu artigo 24, regulamenta que "a verificação do rendimento escolar observará os seguintes critérios: a) avaliação contínua e cumulativa do desempenho do aluno, com prevalência dos aspectos qualitativos sobre os quantitativos e dos resultados ao longo do período sobre os de eventuais provas finais (...)". Assim, compete ao docente a busca de meios para estimular o interesse pelo estudo constante e encontrar maneiras efetivas de subsidiar as avaliações contínuas necessárias.

De fato, a utilização de metodologias ativas de aprendizagem são vistas como uma necessidade em cursos de Odontologia ${ }^{5-7}$ e na área da Saúde ${ }^{8,9}$, sendo inclusive previstas nas Diretrizes Curriculares Nacionais da Odontologia em vigor (Resolução CNE/CES $03 / 02)^{10}$. Uma recente revisão sistemática identificou que a nota de avaliação de estudantes que receberam metodologias ativas de aprendizagem nas áreas de ciências, engenharias e matemática foi cerca de $6 \%$ maior do que estudantes que receberam aulas tradicionais, sendo que estes últimos apresentaram probabilidade 1,5 vezes maior de reprovar em uma avaliação ${ }^{11}$. Além disto, um estudo avaliou o uso do portfólio como um instrumento auxiliar durante o processo de aprendizagem na Odontologia, sendo que este método de avaliação foi considerado excelente ou bom e melhor para a aprendizagem do que as provas convencionais. $^{12}$

Dentre as técnicas de avaliação, algumas proporcionam a aplicação de metodologias ativas de aprendizagem que ultrapassam a barreira da sala de aula. O desafio se torna, então, incentivar o estudante a buscar o aprendizado e se manter estudando também fora da classe em busca de seu próprio aprendizado. Dentre estas técnicas ativas de ensino-aprendizagem e avaliação podem ser citadas o Problem-Based Learning, que apresenta grande potencial para utilização na Odontologia $^{13}$; a "sala de aula invertida", que decorre do conhecimento prévio dos estudantes sobre o conteúdo e tem sido empregada com sucesso no ensino da Periodontia ${ }^{14}$; e a utilização de quiz, que une o potencial avaliativo e a possibilidade de desenvolvimento de metodologias ativas de aprendizagem após sua aplicação, e vem sendo empregado com sucesso na área de ciências biológicas ${ }^{15}$.

Desta maneira, o objetivo deste estudo foi avaliar o efeito da aplicação de quiz no processo de aprendizagem no componente curricular de Periodontia no Curso de Odontologia da Universidade Federal do Paraná (CO/UFPR).

\section{METODOLOGIA}

Este foi um estudo observacional retrospectivo. Foram analisadas as notas da primeira avaliação teórica dos estudantes que cursaram o componente curricular de Periodontia I do CO/UFPR no primeiro e segundo semestres de 2016, com carga horária de 60 horas (30 teóricas e 30 práticas). O projeto de pesquisa foi aprovado pelo Comitê de Ética em Pesquisa em Seres Humanos do Setor de Ciências da Saúde da instituição (Parecer 2.119.596).

\section{Comparação entre grupos "com quiz" e "sem quiz"}

Os estudantes que cursaram Periodontia I no primeiro semestre de 2016 não receberam avaliações semanais por meio da aplicação de quizzes e foram denominados "sem quiz". Com finalidade estritamente didática, a aplicação de 
quiz semanal para aferição do conhecimento dos estudantes e estímulo ao estudo contínuo foi iniciada no segundo semestre de 2016. Portanto, as notas da primeira prova teórica dos estudantes de Periodontia I no segundo semestre de 2016 foram utilizadas e este grupo foi denominado "com quiz". Consideraram-se quizzes perguntas simples (aproximadamente 3) de respostas diretas e curtas (1 a 2 linhas) escritas de maneira livre pelos estudantes ao início de cada aula, contemplando o assunto ministrado na semana anterior. $\mathrm{O}$ estudante respondeu individualmente ao quiz em meia página contendo apenas seu nome e data, sem que fosse necessário copiar a pergunta, a qual foi feita de forma oral e com um tempo aproximado de 2 minutos para a resposta. Nenhum material de apoio pôde ser consultado. Após cada quiz, era realizada uma breve revisão da aula anterior para se criar uma conexão com o assunto do dia. Foram realizados cinco quizzes antes da primeira avaliação teórica considerada neste estudo. Ressalta-se que a primeira avaliação teórica aplicada nos dois semestres de 2016 foi idêntica, possibilitando a comparação entre os dois grupos. As notas da primeira avaliação teórica de cada estudante foram obtidas a partir do diário de classe da turma.

\section{Correlação entre número de quizzes realizados e nota na avaliação teórica}

A fim de se verificar se o estudo contínuo e assiduidade às aulas interferem na nota da avaliação teórica, foi analisada a correlação entre o número de quizzes realizados por cada estudante e sua nota na avaliação teórica. Esta análise foi realizada apenas no grupo "com quiz", computando o número de campos "nota do quiz" preenchidos e "nota da primeira avaliação" do diário de classe. Campos "nota do quiz" em aberto foram descontados do número total de quizzes realizados $(\mathrm{n}=5)$.

\section{Correlação entre nota dos quizzes e da avaliação teórica}

A fim de se verificar se o desempenho no quiz como consequência do estudo contínuo interfere na nota da avaliação teórica, foi analisada a correlação entre a nota dos quizzes realizados por cada estudante e sua nota na avaliação teórica. Esta análise foi realizada apenas no grupo "com quiz", através do acesso aos campos "nota do quiz" e "nota da primeira avaliação" do diário de classe.

\section{Análise estatística}

A distribuição normal dos dados foi testada através do teste de D'Agostino-Pearson K2. As médias e desvio-padrão das notas das primeiras avaliações teóricas foram comparadas por meio do teste $t$ de Student. O teste de correlação de Spearman foi utilizado para se avaliar a correlação entre nota na avaliação teórica e número de quizzes ou nota dos quiz. Todas as análises foram realizadas em software GraphPad Prism 6.0 for Mac OS X (GraphPad Software, Inc., La Jolla, CA, Estados Unidos).

\section{RESULTADOS}

No total, 108 estudantes participaram da pesquisa (53 no grupo "sem quiz" e 55 no grupo "com quiz"). Não houve alunos faltantes na primeira avaliação teórica. No grupo sem quiz, a média $( \pm \mathrm{DP})$ da primeira avaliação teórica foi $69,25 \pm 13,93$, enquanto no grupo com quiz foi $76,75 \pm 16,01 \quad(\mathrm{p}<0,01$; figura 1). Observou-se distribuição normal dos dados $(p>0,05)$ em ambos os grupos, sendo evidente o deslocamento da curva normal à direita no grupo com quiz. (figura 2). Em relação à nota da avaliação no grupo com quiz, houve correlação significativa com o número de quizzes realizados $(\mathrm{r}=0,39$; $\mathrm{p}<0,01)$ e com a nota do quiz $(\mathrm{r}=0,47 ; \mathrm{p}<0,001$; figura 3 ). 


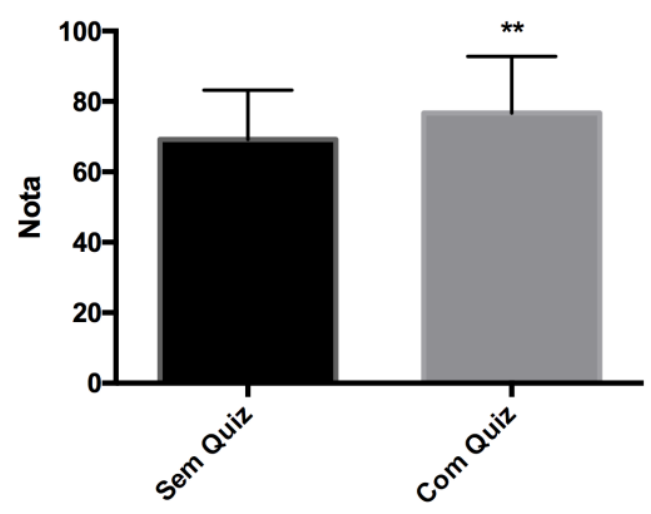

Figura 1: Média ( \pm DP) da nota da avaliação teórica na turma do $1^{\circ}$ semestre (sem quiz) e $2^{\circ}$ semestre (com quiz) de 2016. ** teste $\mathrm{t} ; \mathrm{p}<0.01$
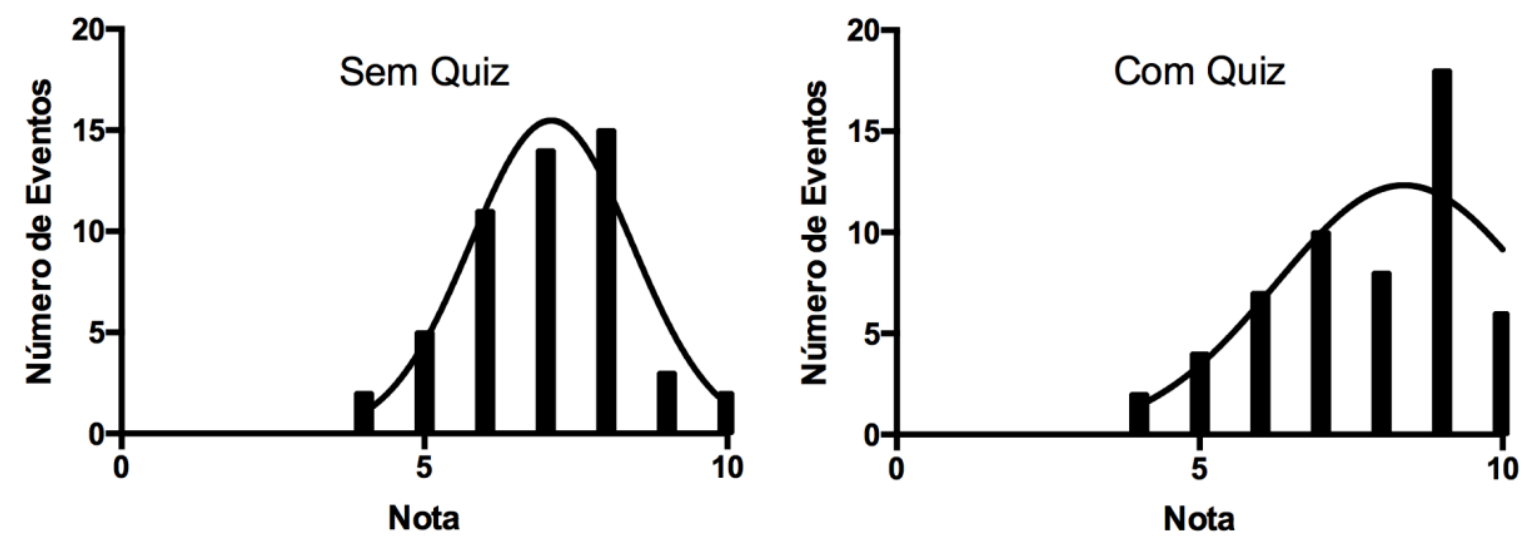

Figura 2: Curva de distribuição das notas da avaliação teórica nas turmas sem quiz e com quiz. Observar deslocamento à direita na curva do grupo com quiz.
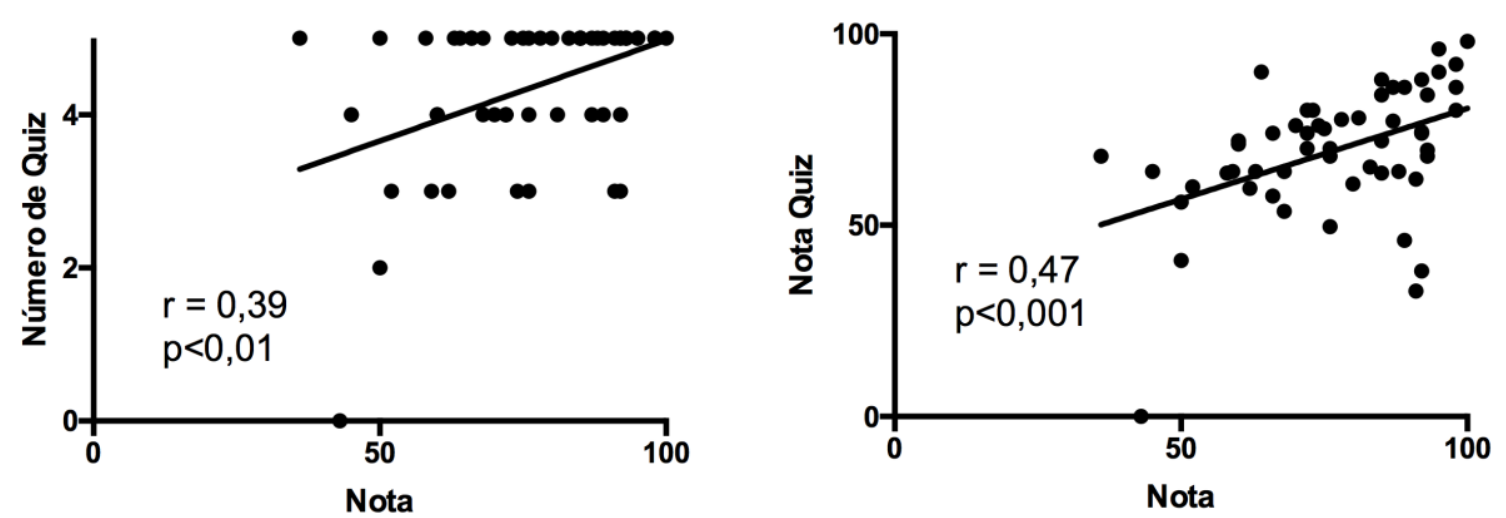

Figura 3: Correlação entre número de quizzes realizados por cada estudante (esquerda) e média da nota do quiz (direita) com a nota da avaliação teórica (eixo x) 


\section{DISCUSSÃO}

Nota-se dificuldade em se exercer práticas participativas, ativas e dialogadas na educação em saúde. Isso pode ser entendido como um reflexo da própria formação acadêmica destes profissionais, na qual as metodologias de ensino e aprendizagem comumente estão centradas no docente $^{16}$. Este estudo retrospectivo avaliou o uso de quizzes ao início de cada aula como forma de estímulo ao estudo semanal e seu reflexo na avaliação teórica no componente curricular de Periodontia I. Os resultados demonstraram um efeito positivo da aplicação de quizzes sobre a nota da avaliação teórica. Esta observação pode ser explicada, além do estímulo à leitura, pelo maior interesse do estudante em sala de aula devido ao uso de metodologias ativas.

De acordo com Carvalho et al., 2016 6 , 67\% de estudantes da graduação e $70 \%$ dos estudantes da pós-graduação em Odontologia relatam nunca terem tido contato com metodologias ativas de aprendizagem. Além disso, a totalidade dos estudantes aprovou o uso de metodologias ativas de aprendizagem após terem sido apresentados às mesmas ${ }^{17}$. Similarmente ao presente estudo, outro trabalho identificou que a nota de avaliação de estudantes que receberam metodologias ativas de aprendizagem foi maior do que estudantes que receberam aulas tradicionais ${ }^{11}$. Por outro lado, um estudo relatou que estudantes do quarto ano do curso de Medicina da Universidade Federal de Goiás preferem aulas tradicionais centradas no docente, sendo que este tipo de aula apresentou significativamente maior frequência de estudantes e porcentagem de acerto nas avaliações $^{8}$.

Apenas a primeira avaliação do semestre foi usada neste estudo. Apesar das avaliações não terem sido entregues aos estudantes (exceto para vista de prova) é possível que se anotem as questões logo após a avaliação e se passe para a turma seguinte, prática esta inibida pelos docentes da disciplina. Como a prova consistiu de questões objetivas e discursivas relacionadas aos conceitos básicos em Periodontia (anatomia do periodonto, etiopatogenia, microbiologia e imunologia das doenças periodontais, epidemiologia e fatores modificadores da resposta do hospedeiro), dificilmente houve registro preciso das questões ou de suas respostas.

A avaliação por meio de quiz semanal não substituiu a avaliação teórica no contexto apresentado, mas contribuiu com incremento de $25 \%$ para a nota final do estudante referente a este primeiro módulo. Os outros $75 \%$ foram derivados da nota teórica. A avaliação por quiz apresenta as vantagens de contribuir para a transmissão formativa do conhecimento, pois permite ao docente saber as dificuldades dos estudantes e assim fornecer subsídios para que as mesmas sejam enfrentadas em tempo hábil; descentralizar o processo avaliativo, permitindo a aferição dos estudos de maneira continuada e não em um único momento; além de incentivar a leitura e estudo constantes.

Devido à entrada semestral dos estudantes no $\mathrm{CO} / \mathrm{UFPR}$, ingressantes em um único concurso vestibular, poderia ser levantada a hipótese de que aqueles que ingressam no primeiro semestre apresentem melhores notas em avaliações em comparação com os ingressantes no segundo semestre. Porém, a Periodontia I é ministrada no quinto período do curso, e os resultados mostraram que os ingressantes no segundo semestre (exceto pelos estudantes eventualmente não seguindo a periodização recomendada) obtiveram melhores notas de avaliação que os ingressantes no primeiro semestre. Ressalta-se que este estudo é retrospectivo e não teve como objetivo avaliar os dados relacionados aos estudantes, como sexo, idade, forma de ingresso no vestibular, entre outros; apenas as suas notas. Por este motivo não 
houve pesquisa de satisfação relacionada a esta forma de ensino. Outros estudos prospectivos poderiam melhor elucidar a satisfação dos estudantes e observar perfis sociodemográficos de preferência por metodologias ativas.

\section{CONCLUSÃO}

Concluiu-se que o uso de quiz no ensino da Periodontia pode se configurar como técnica de estímulo à aprendizagem e refletir positivamente no conhecimento dos estudantes.

\section{ABSTRACT \\ Continuous learning evaluation through the application of weekly quiz in Periodontics.}

Continuous and routine evaluation keeps the student studying, reading and learning permanently, as well as lightens the impact of formal theoretical exams on the traditional criteria for passing a course. The aim of this study was to evaluate the impact of weekly quiz application on the formal theoretical exam score in the course of Periodontics I - a theoreticalpractical course - at the School of Dentistry at Federal University of Paraná, Brazil. For this retrospective observational study, the fields "quiz score" and "exam score" of the register book from students of the first and second term of 2016, "no quiz" (n=53) and "weekly quiz application" ( $(n=55)$, respectively, were analyzed. Formal theoretical exams were identical for both students groups. Data related to the number of quizzes taken by each student and quiz scores were tabulated. Student's t-test and Spearman correlation coefficient test were used for statistical analyses. Weekly quiz application moved the theoretical exam scores curve to the right and scores were significantly greater in this group ( $\mathrm{p}<0.01)$. Besides, there was a significant correlation between theoretical exam score and the number of quizzes taken by each student $(\mathrm{r}=0.39 ; \mathrm{p}<0.01)$, as well as with mean quiz score $(\mathrm{r}=0.47 ; \mathrm{p}<0.01)$. We concluded that weekly application of quiz as an evaluation instrument in Periodontics positively influences acquired knowledge, probably due to stimulating permanent studying.

Descriptors: Educational Measurement. Learning. Periodontics. Observational Study. Education, Dental.

\section{REFERÊNCIAS}

1. Kenski VMA. Vivência escolar dos estagiários e a prática de pesquisa em estágios supervisionados. In: Piconez SCB, org. A prática de ensino e o estágio supervisionado. 2. ed. Campinas: Papirus; 1994.

2. Luz AA. A avaliação no ensino superior. Educ Rev. 1997; 13:55-66.

3. Marquesin DFB, Benevides CR. Avaliação da aprendizagem no ensino superior. Reflexões sobre a "cola". Rev Educ. 2011; 18:9-18.

4. Lei 9.394, de 20 de dezembro de 1996. Presidência da República. [Acesso em 17 mar. 2017]. Disponível em: http://www. planalto.gov.br/ccivil_03/leis/L9394.htm.

5. Matias KK. Metodologias de ensino e práticas pedagógicas em um curso de graduação em odontologia [tese de doutorado]. Universidade Federal de Goiás, 2013. 132 pp.

6. Carvalho WM, Cawahisa PT, Scheibel PC, Botelho JN, Terada RSS, Rocha NB, et al. Aceitação da utilização de metodologias ativas nos estágios no SUS por discentes da graduação e pós-graduação em odontologia. Rev ABENO. 2016;16:88-98.

7. Reul MA, Lima ED, Irineu KN, Lucas RSCC, Costa EMMB, Madruga RCR. Metodologias ativas de ensino aprendizagem na graduação em odontologia e a contribuição da monitoria - relato de experiência. Rev ABENO. 2016;16:62-8.

8. De Deus JM, Nonato DR, Alves RRF, Silva MMM, Amaral AF, Bollela VR. Aula centrada no aluno versus aula centrada no professor. Desafios para mudança. Rev Bras Educ Méd. 2014; 58:419-26.

9. Freitas CM, Freitas CASL, Parente JRF, 
Vasconcelos MIO, Lima GK, Mesquita KO, et al. Uso de metodologias ativas de aprendizagem para a educação na saúde: análise da produção científica. Trab Educ Saúde. 2015;13:117-30.

10. Resolução CNE/CES 3, de 19 de fevereiro de 2002. Conselho Nacional de Educação e Câmara de Educação Superior. [Acesso em 17 mar. 2017]. Disponível em: http://portal.mec. gov.br/cne/arquivos/pdf/CES032002.pdf.

11. Freeman S, Eddy SL, McDonough M, Smith MK, Okoroafor $\mathrm{N}$, Jordt $\mathrm{H}$, et al. Active learning increases student performance in Science, engineering, and mathematics. Proc Natl Acad Sci USA. 2014; 111:8410-15.

12. Frota MMA, Menezes LMB, Alencar CH, Jorge LS, Almeida MEL.O portfólio como estratégia facilitadora do processo de ensinoaprendizagem para a formação em odontologia. Adequação de metodologias de ensino utilizando o ambiente virtual de aprendizagem. Rev ABENO. 2011;11:23-8.

13. Saliba NA, Moimaz SAS, Chiaratto RA, Tiano AVP. A utilização da metodologia PBL em odontologia: descortinando novas possibilidades ao processo ensinoaprendizagem. Rev Odonto Ciênc. 2008;23: 392-6.
14. Santos FA, Castro FF, Moura ME, Pochapski MT, Pilatti GL. Metodologias de sala de aula invertida no ensino de Periodontia [resumo]. Rev ABENO. 2016;16(supl 2):54.

15. Silva JMA, Canedo RV, Abrantes TAS, Santos RT, Souza RA, Utagawa CY. Quiz: um questionário eletrônico para autoavaliação e aprendizagem em genética e biologia molecular. Rev Bras Educ Méd. 2010;34:60714.

16. Vasconcelos EM. Educação popular: de uma prática alternativa a uma estratégia de gestão participativa das políticas de saúde. Physis (Rio J). 2004; 14:67- 83.

17. Noro LRA, Farias-Santos BCS, Sette-deSouza PH, Pinheiro IAG, Borges REA, Nunes LMF, et al. O professor (ainda) no centro do processo ensino-aprendizagem em Odontologia. Rev ABENO. 2015;15:2-11.

\section{Correspondência para:}

Prof. Dr. João Paulo Steffens

e-mail: joao.steffens@ufpr.br

Universidade Federal do Paraná

Departamento de Estomatologia

Av. Pref. Lothário Meissner, 632

80210-170 Curitiba/PR 